ARTICLE

Received 21 Jan 2015 | Accepted 4 Jun 2015 | Published 9 Jul $2015 \quad$ DOl: 10.1038/ncomms8755 OPEN

\title{
Vibronic origin of long-lived coherence in an artificial molecular light harvester
}

James Lim ${ }^{1, \star}$, David Paleček ${ }^{2,3, \star}$, Felipe Caycedo-Soler ${ }^{1}$, Craig N. Lincoln ${ }^{4}$, Javier Prior ${ }^{5}$, Hans von Berlepsch ${ }^{6}$, Susana F. Huelga1, Martin B. Plenio', Donatas Zigmantas ${ }^{2} \&$ Jürgen Hauer ${ }^{4}$

Natural and artificial light-harvesting processes have recently gained new interest. Signatures of long-lasting coherence in spectroscopic signals of biological systems have been repeatedly observed, albeit their origin is a matter of ongoing debate, as it is unclear how the loss of coherence due to interaction with the noisy environments in such systems is averted. Here we report experimental and theoretical verification of coherent exciton-vibrational (vibronic) coupling as the origin of long-lasting coherence in an artificial light harvester, a molecular J-aggregate. In this macroscopically aligned tubular system, polarization-controlled 2D spectroscopy delivers an uncongested and specific optical response as an ideal foundation for an in-depth theoretical description. We derive analytical expressions that show under which general conditions vibronic coupling leads to prolonged excited-state coherence.

\footnotetext{
${ }^{1}$ Institut für Theoretische Physik, Universität Ulm, Albert-Einstein Allee 11, 89069 Ulm, Germany. ${ }^{2}$ Department of Chemical Physics, Lund University, PO Box 124, SE-22100 Lund, Sweden. ${ }^{3}$ Department of Chemical Physics, Charles University in Prague, Ke Karlovu 3, 12116 Praha 2, Czech Republic. 4 Photonics Institute, Vienna University of Technology, Gusshausstrasse 27, 1040 Vienna, Austria. ${ }^{5}$ Departamento de Física Aplicada, Universidad Politécnica de Cartagena, Cartagena 30202, Spain. ${ }^{6}$ Forschungszentrum für Elektronenmikroskopie, Institut für Chemie und Biochemie, Freie Universität Berlin, Fabeckstraße 36a, D-14195 Berlin, Germany. ${ }^{*}$ These authors contributed equally to this work. Correspondence and requests for materials should be addressed to J.H. (email: juergen.hauer@tuwien.ac.at).
} 
T he remarkably high efficiency in photosynthesis, where nine out of ten absorbed photons reach the reaction centre, is a fascinating field of modern research. In such photosynthetic complexes, structure, dynamics and function are inextricably linked. A conserved building block comprises strongly absorbing pigments arranged in close proximity to one another supported by the surrounding protein scaffold ${ }^{1,2}$. Typical inter-pigment distances are of order of $10 \AA$ and photon absorption leads to the formation of delocalized excited electronic states (excitons) shared by two or more pigment molecules. Exciton creation, migration and trapping are central to the functionality of a photosynthetic apparatus. The controlled and adjustable arrangement of the pigments tunes the electronic network and the properties of its interaction with the vibrational environment that is associated with either the pigments or the protein. The detailed balance of these properties determines the efficiency of light-harvesting systems $s^{3,4}$.

Exciton dynamics can be efficiently probed by two-dimensional (2D) electronic spectroscopy ${ }^{5}$. This technique revealed oscillatory signals in the spectral response of a wide variety of photosynthetic aggregates ${ }^{6,7}$. Initially ascribed to excitonic beatings, oscillations have been found to persist up to several hundreds of femtoseconds at room temperature ${ }^{8-10}$. This timescale exceeds typical dephasing rates in the condensed phase and becomes comparable to exciton transfer times ${ }^{1}$, thus posing the question of the nature and functional relevance of these coherences ${ }^{4}$. Unfortunately, the complex structure of $2 \mathrm{D}$ signals makes the unambiguous identification of the underlying mechanisms that support such long-lived coherences a challenging task and several hypotheses to explain them have been formulated ${ }^{11-21}$. The different approaches can be classified into theories including coherent interaction of excitons with intra-pigment vibrations ${ }^{11-15}$ and theories focusing on incoherent exciton-protein interaction such as correlated fluctuations $s^{16-18}$. It is possible that some of these mechanisms may coexist on certain timescales and that one or another may become dominant depending on the system under consideration.

In this work, we show that the relatively simple excitonic structure of a molecular J-aggregate provides an ideal test case to identify the microscopic mechanism behind long-lived oscillations in electronic $2 \mathrm{D}$ signals. The investigated J-aggregate is tubular and aligns along the sample's flow direction when in solution. In addition, the J-aggregate exhibits excitonic bands with roughly orthogonal transition dipole moments. It is this combination of perpendicular excitonic transitions and macroscopic alignment that makes electronic 2D spectroscopy with polarization-controlled excitation pulses an ideal tool to study coherence effects between the excitonic bands. This approach significantly reduces the complexity of retrieved 2D signals, leading to only two peaks with oscillatory components in specific regions of the $2 \mathrm{D}$ maps, that is, one on the diagonal and one as a cross-peak for non-rephasing and rephasing signal components, respectively. Employing a vibronic model, we derive analytical expressions that show how system parameters such as electronic decoherence rates and exciton-vibrational resonance determine the amplitude and lifetime of oscillatory signals. Fitting the analytical expressions to measured data, the vibronic model achieves quantitative agreement with experimental observations. Concerning potential functional relevance of the observed oscillations, we show that the long-lived oscillatory signals in our system are dominated by excited-state coherence rather than ground-state coherence.

\section{Results}

The system. J-aggregates of cyanine dyes are promising candidates for artificial antenna systems ${ }^{2-26}$. They are chemically versatile and self-assemble into various extended supramolecular structures in aqueous solution ${ }^{27}$. Here a system that can be considered a macroscopically aligned synthetic light harvester was studied, namely a molecular J-aggregate of $\mathrm{C} 8 \mathrm{O} 3$-monomers whose aggregation behaviour is well known ${ }^{28,29}$. As revealed by cryogenic transmission electron microscopy ${ }^{30}$, the aggregate structure is best described as a double-layered nanotube with outer diameter $\sim 11 \mathrm{~nm}$ and lamellar spacing of $\sim 2.2 \mathrm{~nm}$ between the chromophore layers. In addition, superhelical bundles of these tubes can also form though the addition of polyvinyl alcohol inhibits this process and thereby avoids singlelayered tube formation ${ }^{24}$ and maintains a stable solution over several weeks ${ }^{31}$. A drawing of the J-aggregate under investigation, from here on referred to as $\mathrm{C8O} 3$, is shown in Fig. 1a. The bilayer configuration of $\mathrm{C} 8 \mathrm{O} 3$ allows the effect of different decoherence rates to be studied as the outer solvent-exposed layer shows faster decoherence than the inner protected layer.

The structural properties of the aggregate are remarkable: the 11-nm outer diameter is contrasted by a length of several micrometres. Circulating solvated $\mathrm{C} 8 \mathrm{O} 3$ with a wire-guided jet (Fig. 1a) leads to a macroscopic orientation of the tubes because
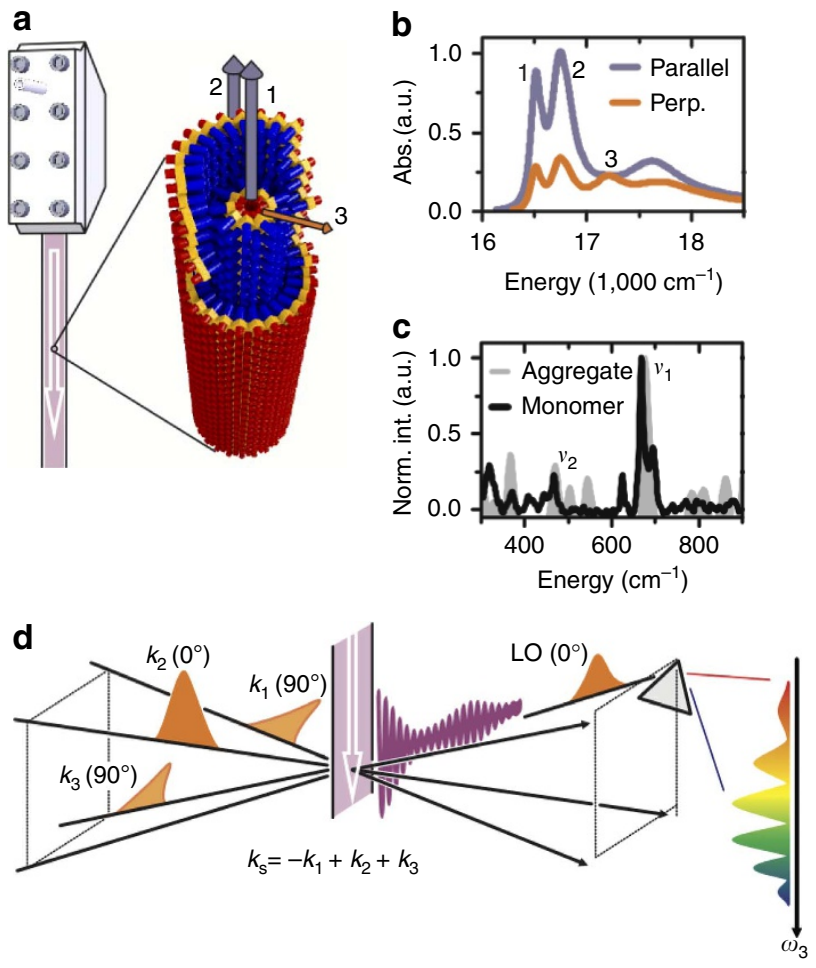

Figure 1 | C803 and polarization-controlled 2D spectroscopy. (a) Wireguided window-free jet used for sample circulation, along with a schematic of the double-layered structure of the $\mathrm{C} 8 \mathrm{O} 3$ aggregate. The aggregates align along the flow direction (white arrow). The transition dipole directions of bands 1-3 are displayed by arrows, which are mainly polarized along the tube axis (bands 1 and 2 shown in blue) or perpendicular to the axis (band 3 shown in orange). (b) Absorption spectra in arbitrary units, Abs. (a. u.), with light polarized parallel (blue) and perpendicular (perp.; orange) to the flow direction. (c) Non-resonant Raman spectra of the $\mathrm{C} 8 \mathrm{O} 3$ monomer (black line) and aggregate (grey area). The vibrational frequencies $v_{1}$ and $v_{2}$ are close to the exciton energy splitting between bands 1 and 3 and bands 2 and 3 , respectively. (d) Polarization-controlled 2D spectroscopy with three excitation pulses ( $k_{1}$ to $k_{3}$ ) and a local oscillator (LO) for heterodyne detection of the signal field, depicted as an oscillating line. Polarization orientation $\left(0^{\circ}\right.$ or $\left.90^{\circ}\right)$ is given with respect to the longitudinal axis of aligned $\mathrm{C} 8 \mathrm{O} 3$. 
the longitudinal axis preferentially aligns along the flow direction. This creates anisotropy for linearly polarized light, as shown in Fig. 1b. Linear dichroism measurements ${ }^{31}$ and redox-chemistry studies $^{32}$ assign bands 1 and 2 to longitudinal transitions localized on the inner and outer cylinders, respectively (Fig. 1a). Transitions to band 3 are preferentially polarized perpendicular to the long axis of $\mathrm{C} 8 \mathrm{O} 3$ and are shared by both layers. A detailed description of sample-preparation methods and band assignments is given in the Supplementary Notes 1 and 2 .

Fitting the well-defined absorption peaks of $\mathrm{C} 8 \mathrm{O} 3$ with Lorentzian functions (see Supplementary Note 2) reveals an exciton energy difference between bands 1 and 3 of $\Delta \Omega_{31} \approx 690 \mathrm{~cm}^{-1}$ and $\Delta \Omega_{32} \approx 460 \mathrm{~cm}^{-1}$ for bands 2 and 3 . Both exciton energy splittings are close to vibrational frequencies $v_{1} \approx 668 \mathrm{~cm}^{-1}$ and $v_{2} \approx 470 \mathrm{~cm}^{-1}$ observed in non-resonant Raman spectra $^{33}$ (Fig. 1c). These vibrational frequencies are measured in both the monomer and aggregate Raman spectra, that is, they are not aggregation-induced Raman bands. Strongly enhanced modes at similar energies were observed in resonant Raman spectra of a related cyanine dye, and can be assigned to out-of-plane vibrations ${ }^{34}$. Such out-of-plane vibrations were shown to couple strongly to excitons ${ }^{35}$. The quasi-resonance between the vibrational frequencies $v_{1}$ and $v_{2}$ and exciton energy splittings $\Delta \Omega_{31}$ and $\Delta \Omega_{32}$ provides us with an interesting scenario of possible coherent interaction between bands (excitons) and vibrations ${ }^{11,13,14,21,36}$. Such exciton-vibrational coupling induces vibronic $^{12}$ and vibrational coherences ${ }^{15}$, which can both lead to long-lived beating signals in $2 \mathrm{D}$ spectra. Here we emphasize that coherence in the electronic excited-state manifold is referred to as vibronic and in the ground-state manifold as vibrational. Identifying the dominant contribution is of fundamental importance because only vibronic coherence, which manifests in excited-state dynamics, can enhance exciton transport and thus support light-harvesting function ${ }^{37-39}$.

Experimental results. The absorption spectrum of a light-harvesting system may be heavily congested because of overlapping excitonic bands and the resulting $2 \mathrm{D}$ signal would exhibit significant overlap between diagonal and cross-peaks, thereby impeding further analysis. It has been suggested to employ laser pulses of different relative polarization to selectively address relevant excitation pathways to obtain a clearer $2 \mathrm{D}$ signal ${ }^{40}$. However, the advantage of polarization-controlled 2D spectroscopy has been limited by the isotropic nature of the investigated samples (an ensemble). In the experiment presented here, these problems are circumvented by the measurement of the macroscopically aligned $\mathrm{C} 8 \mathrm{O} 3$. The transition dipole moments of bands 1 and 2 are preferentially parallel to the longitudinal axis while band 3 is orthogonal, thus allowing for optimal polarization selectivity. This combination reduces the obtained 2D maps to only two relevant peaks with negligible overlap and an up to 30 times stronger signal intensity as compared with the isotropic case $^{41}$

The ideal pulse sequence to isolate beating signals between states with orthogonal transition dipole moments, that is, bands 1 and 3 in the present case, is depicted in Fig. 1d, where the phasematched direction for measuring rephasing spectra is displayed: non-rephasing spectra can be measured along the same phasematched signal direction by changing the order of the first two pulses (see Methods). After subtraction of the non-oscillatory background, we performed a Fourier transformation along waiting time $t_{2}$ for all points on the $2 \mathrm{D}\left(\omega_{1}, \omega_{3}\right)$ map. The resulting $\omega_{2}$ plots allow the lineshape of beating signal with frequency $\omega_{2}$ to be visualized as a function of position in $\left(\omega_{1}, \omega_{3}\right)$ space. The slice at the exciton energy splitting between bands 1 and $3\left(\omega_{2}=705 \pm 20 \mathrm{~cm}^{-1}\right.$ with the experimental resolution of $\left.\pm 20 \mathrm{~cm}^{-1}\right)$ reveals a non-rephasing diagonal peak N11 and a rephasing cross-peak R31 as shown in Fig. 2a,b, respectively. N11 is centred at $\left(\omega_{1}, \omega_{3}\right)=\left(\Omega_{1}, \Omega_{1}\right)$ with exciton energy $\Omega_{1} \approx 16,405 \mathrm{~cm}^{-1}$ of band 1 and a symmetric linewidth $2 \Gamma_{g 1} \approx 130 \mathrm{~cm}^{-1}$ along both $\omega_{1}$ and $\omega_{3}$ axes (Fig. 2a). The centre of R31 is located at $\left(\omega_{1}, \omega_{3}\right)=\left(\Omega_{3}, \Omega_{1}\right)$ with exciton energy $\Omega_{3} \approx 17,125 \mathrm{~cm}^{-1}$ of band 3 and asymmetric linewidths $2 \Gamma_{g 3} \approx 300 \mathrm{~cm}^{-1}$ and $2 \Gamma_{g 1} \approx 130 \mathrm{~cm}^{-1}$ along $\omega_{1}$ and $\omega_{3}$ axes, respectively (Fig. 2b). In peak amplitude, R31 is $\sim 30 \%$ of N11.
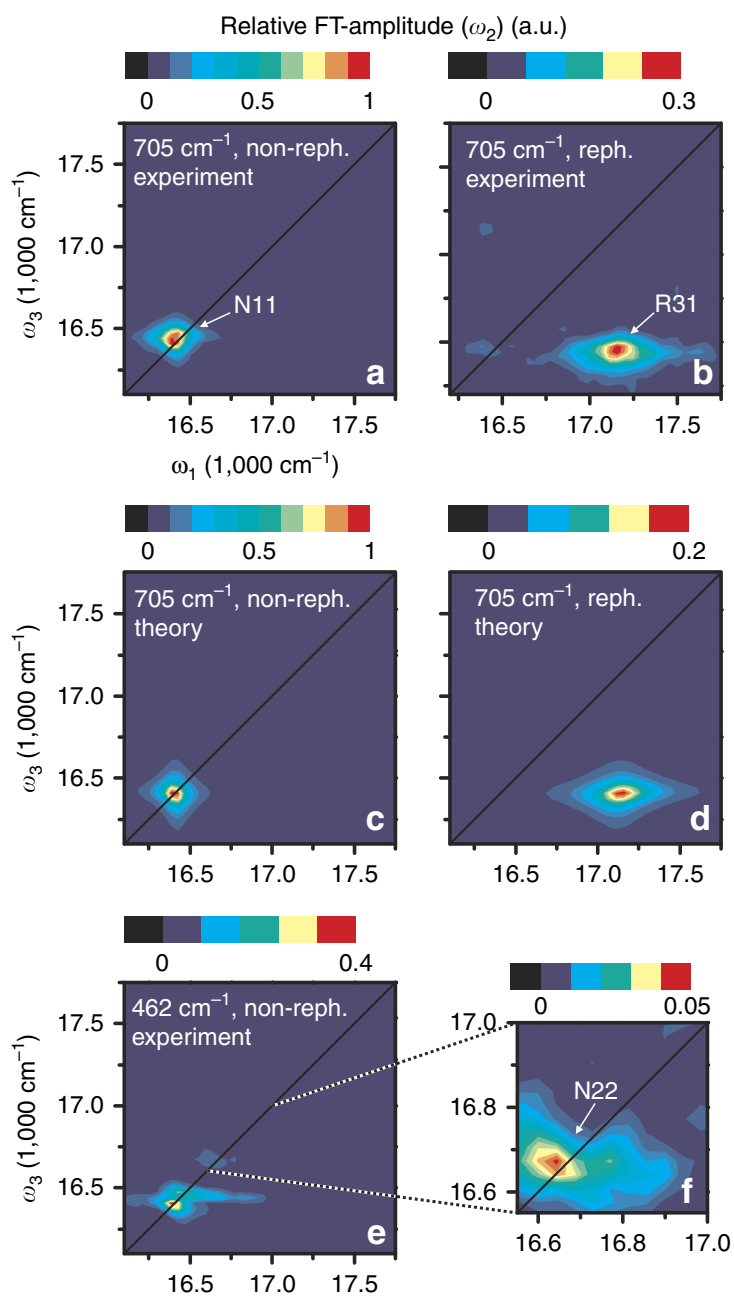

Figure 2 | Experimental and theoretical 2D spectra. $(\mathbf{a}, \mathbf{b})$ The Fouriertransform (FT) amplitude maps of non-rephasing (non-reph.) and rephasing (reph.) spectra at $\omega_{2}=705 \pm 20 \mathrm{~cm}^{-1}$, which reveal the presence of a non-rephasing diagonal peak N11 and a rephasing cross-peak R31. These peaks stem from the coherent interaction of bands 1 and 3 with the quasi-resonant vibrational mode with frequency $v_{1} \approx 668 \mathrm{~cm}^{-1}$. The amplitude of N11 is about three times larger than R31. The lineshape of N11 is symmetric along both $\omega_{1}$ and $\omega_{3}$ axes, while that of R31 is elongated along $\omega_{1}$ axis. (c,d) The simulated spectra at $\omega_{2}=705 \mathrm{~cm}^{-1}$ with $\mathrm{N} 11$ and R31. (e) The FT amplitude map at $\omega_{2}=462 \pm 20 \mathrm{~cm}^{-1}$ reveals coherent interaction of bands 2 and 3 with the quasi-resonant vibrational mode with frequency $v_{2} \approx 470 \mathrm{~cm}^{-1}$. However, as depicted in (f) the associated nonrephasing peak $\mathrm{N} 22$ at $\omega_{1,3} \approx 16,670 \mathrm{~cm}^{-1}$ is weak and only amounts to $5 \%$ of $\mathrm{N} 11$ at $\omega_{2}=705 \pm 20 \mathrm{~cm}^{-1}$ (see a). The diagonal peak at $\omega_{1,3} \approx 16,400 \mathrm{~cm}^{-1}$ in e stems from $\mathrm{N} 11$, with a peak centred at $\omega_{2}=705 \pm 20 \mathrm{~cm}^{-1}$, but broad enough to appear at $\omega_{2}=462 \pm 20 \mathrm{~cm}^{-1}$. All measurements were carried out at room temperature. 
Turning to the $\omega_{2}$ slice corresponding to the energy splitting between bands 2 and $3\left(\omega_{2}=462 \pm 20 \mathrm{~cm}^{-1}\right)$, Fig. 2e, $\mathrm{f}$ reveal a diagonal non-rephasing peak $\mathrm{N} 22$, which is centred at $\left(\omega_{1}, \omega_{3}\right)=\left(\Omega_{2}, \Omega_{2}\right)$ with the exciton energy $\Omega_{2} \approx 16,672 \mathrm{~cm}^{-1}$ of band 2 and a symmetric linewidth $2 \Gamma_{g 2} \approx 225 \mathrm{~cm}^{-1}$ along $\omega_{1}$ and $\omega_{3}$ axes. The amplitude of N22 is only $5 \%$ of N11.

Theoretical model. To describe the long-lived oscillations in $\mathrm{N} 11$ and R31, a vibronic model is employed that describes the coupling of bands 1 and 3 to a quasi-resonant vibrational mode with frequency $v_{1}$. Consider a system with electronic ground state $\left|g_{k}\right\rangle$ and excited states for bands 1 and 3 , denoted by $\left|1_{k}\right\rangle$ and $\left|3_{k}\right\rangle$, respectively, where $k=0$ and 1 denote the vibrational ground and excited states, respectively (Fig. 3a). The vibronic coupling between the quasi-resonant states $\left|3_{0}\right\rangle$ and $\left|1_{1}\right\rangle$ leads to unnormalized vibronic eigenstates $\left\langle\tilde{3}_{0}\right|=\left\langle 3_{0}\right|+\epsilon\left\langle 1_{1}\right|$ and $\left\langle\tilde{1}_{1}\right|=\left\langle 1_{1}\right|-\epsilon\left\langle 3_{0}\right|$. Here $\epsilon$ represents the degree of vibronic mixing defined by

$$
\epsilon=i v_{1} \sqrt{S_{1}}\left(i \Delta v_{1}-\Gamma_{13}\right)^{-1}
$$

where $\Delta v_{1}=\left(\Omega_{3}-\Omega_{1}\right)-v_{1}$ denotes the detuning between $\left|3_{0}\right\rangle$ and $\left|1_{1}\right\rangle$, that is, between the exciton energy splitting and vibrational frequency, and $S_{1}$ denotes the Huang-Rhys factor of the vibrational mode, which in turn quantifies the strength of the vibronic coupling (see Supplementary Note 2 for details of the derivation). The electronic decoherence rate $\Gamma_{g k}$ describes the exponential decay rate of the coherence between electronic ground state and band $k$, while $\Gamma_{13}$ represents the overall exponential decay rate of the inter-exciton coherence between bands 1 and 3. In our model, we do not consider inhomogeneous broadening, which is justified by the observation that the experimentally measured absorption spectrum is well matched to a sum of Lorentzian functions with the linewidths $2 \Gamma_{g k}$ (see Supplementary Note 2). This is valid when homogeneous broadening dominates the linewidths and the Huang-Rhys factors are sufficiently small, as is the case here. In addition, the lineshape of N11 (Fig. 2a) is not elongated along the diagonal $\omega_{1}=\omega_{3}$, implying our $2 \mathrm{D}$ signal is dominated by homogeneous broadening. The same conclusion is reached from analysing $2 \mathrm{D}$ correlation spectra ${ }^{33}$.

In nonlinear spectroscopy, the molecular response to laser excitation is described by response functions ${ }^{42}$. According to the vibronic model described above, the response function for the oscillatory signals in N11 reads

$$
\mathcal{R}_{\mathrm{N} 11}=\mu_{1}^{2} \mu_{3}^{2} \Gamma_{g 1}^{-2}\left(e^{\left[i\left(\Delta \Omega_{31}+\delta \omega\right)-\Gamma_{13}\right] t_{2}}+e^{\left[i\left(v_{1}-\delta \omega\right)-\gamma_{v}\right] t_{2}} \epsilon^{2}\right),
$$

with $\mu_{1}$ and $\mu_{3}$ denoting the transition dipole moment of bands 1 and 3 , respectively. The prefactor $\Gamma_{g 1}^{-2}$ stems from the lineshape of N11, $\gamma_{v}$ denotes the dissipation rate of the vibrations and $\delta \omega$ stands for the frequency shift of the vibronic eigenstates $\left\langle\tilde{3}_{0}\right|$ and $\left\langle\tilde{1}_{1}\right|$ relative to the uncoupled states $\left\langle 3_{0}\right|$ and $\left\langle 1_{1}\right|$ due to the vibronic coupling (see Fig. $3 \mathrm{a}$ and Supplementary Note 2 for further details). The coupling was found to be sufficiently strong to induce non-negligible vibronic mixing $|\epsilon|^{2} \approx 0.03$, which leads to a long-lived beating signal in N11 up to $t_{2} \approx 800 \mathrm{fs}$, as shown in Fig. 3b. These results imply that the initial excitonic part of $\left|1_{0}\right\rangle\left\langle\tilde{3}_{0}\right|$ decays rapidly with $1 / e$ decay time of $\Gamma_{13}^{-1} \approx 66 \mathrm{fs}$, while the vibronic coherence $\left|1_{0}\right\rangle\left\langle\tilde{1}_{1}\right|$ explains a long-lived oscillatory signal in N11: here $\left|1_{0}\right\rangle\left\langle\tilde{3}_{0}\right|\left(\left|1_{0}\right\rangle\left\langle\tilde{1}_{1}\right|\right)$ represents coherence between two vibronic states $\left|1_{0}\right\rangle$ and $\left\langle\tilde{3}_{0}\right|\left(\left|1_{0}\right\rangle\right.$ and $\left.\left\langle\tilde{1}_{1}\right|\right)$, respectively. a
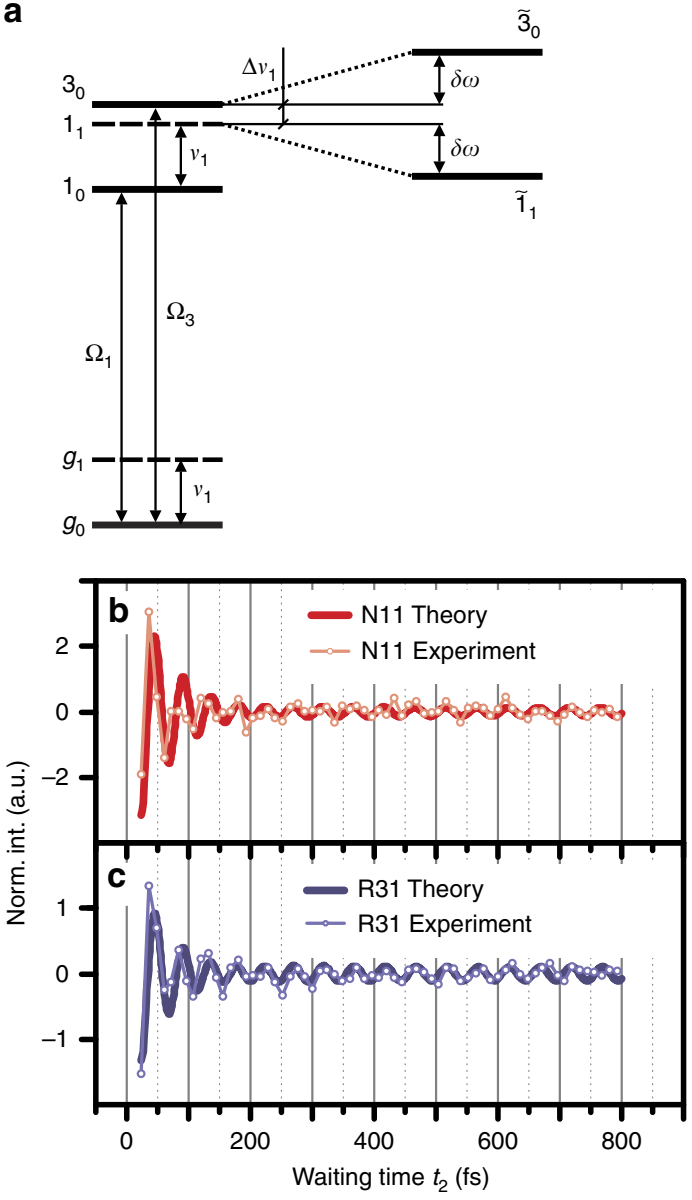

Figure 3 | Vibronic model. (a) We consider a vibronic model for bands 1 and 3 coupled to a vibrational mode with frequency $v_{1} \approx 668 \mathrm{~cm}^{-1}$ (see Supplementary Note 2). The vibronic states $\left|k_{0}\right\rangle$ and $\left|k_{1}\right\rangle$ denote the vibrational ground and first excited states of an electronic state $|k\rangle$, respectively, with the single index states $|g\rangle,|1\rangle$ and $|3\rangle$ denoting the electronic ground state and bands 1 and 3, respectively. The exciton energy splitting $\Delta \Omega_{31}=\Omega_{3}-\Omega_{1}$ between bands 1 and 3 is quasi-resonant with the vibrational frequency $v_{1}$, where the detuning is denoted by $\Delta v_{1}=\Delta \Omega_{31}-v_{1}$. The exciton-vibrational coupling between uncoupled states $\left|3_{0}\right\rangle$ and $\left|1_{1}\right\rangle$ leads to vibronic eigenstates $\left|\tilde{3}_{0}\right\rangle$ and $\left|\tilde{1}_{1}\right\rangle$, each of which is a superposition of $\left|3_{0}\right\rangle$ and $\left|1_{1}\right\rangle$, leading to an energy-level shifting by $\delta \omega$. (b) The time trace of $\mathrm{N} 11$ in normalized intensity (Norm. Int.) against waiting time $t_{2}$, where the experimental results are shown as light red circles and the theoretical simulation is shown as a full red line. (c) The time trace of R31 where the experimental results are shown as light blue circles and the simulated data are depicted as a full blue line. The root mean squared deviation between the experimental results and theoretical simulation in $\mathbf{b}$ and $\mathbf{c}$ is 0.92 and 0.59 , respectively.

The response function for the oscillatory contributions to R31 is given by

$$
\begin{aligned}
\mathcal{R}_{\mathrm{R} 31}= & \mu_{1}^{2} \mu_{3}^{2} \Gamma_{g 3}^{-1} \Gamma_{g 1}^{-1} \\
& \times\left(e^{\left[i\left(\Delta \Omega_{31}+\delta \omega\right)-\Gamma_{13}\right] t_{2}}+e^{\left[i\left(v_{1}-\delta \omega\right)-\gamma_{v}\right] t_{2}} \epsilon^{2}\left(\eta_{e}-\eta_{g}\right)\right),
\end{aligned}
$$

where $\Gamma_{g 3}^{-1} \Gamma_{g 1}^{-1}$ derives from the asymmetric lineshape of R31 (see Fig. $2 \mathrm{~b}, \mathrm{~d}$ ). Here $\eta_{e}$ and $\eta_{g}$ represent the contribution of excited-state vibronic coherence $\left|1_{0}\right\rangle\left\langle\tilde{1}_{1}\right|$ and ground-state vibrational coherence $\left|g_{0}\right\rangle\left\langle g_{1}\right|$, respectively, to the long-lived beating signal in R31 (see Supplementary Note 2). The vibrational coherence in the electronic ground-state manifold does not play a role in exciton transfer dynamics, but nonetheless modulates the 
2D spectra. A fit of model parameters to experimental results (Fig. 3c) shows that $\left|\eta_{e}\right| \approx 2.5\left|\eta_{g}\right|$. This means the long-lived beating signal in R31 is dominated by the excited-state coherence $\left|1_{0}\right\rangle\left\langle\tilde{1}_{1}\right|$. The short-lived beating signal in R31 is induced by $\left|1_{0}\right\rangle\left\langle\tilde{3}_{0}\right|$, as is the case for N11. We note that the signal at N11, with approximately three times the amplitude of R31, is exclusively determined by excited-state contributions. Details of this vibronic model and the corresponding Feynman diagrams for the spectral components $\mathrm{N} 11$ and R31 are discussed in the Supplementary Note 2 .

These results demonstrate how an excitonic system within a noisy environment can exhibit long-lasting coherent features: the observed long-lived oscillations are the result of coherent interaction of excitonic bands with an underdamped, quasiresonant vibration. This vibronic mechanism requires the vibrational dissipation rate $\gamma_{v}$ to be much slower than the electronic decoherence rate $\Gamma_{13}$, which is the case for $\mathrm{C} 8 \mathrm{O} 3$, where $\gamma_{v} \lesssim(1 \mathrm{ps})^{-1}$ and $\Gamma_{13} \approx(66 \mathrm{fs})^{-1}$. The difference in electronic and vibrational decoherence rates can be rationalized from the fact that excitons and vibrations are related to the motion of electrons and nuclei, respectively. The lower mass of electrons as compared with nuclei makes excitons more mobile and therefore more sensitive to environmental fluctuations, such as local electric fields, than vibrations. We note that the vibronic mixing leading to long-lived beating signals in $2 \mathrm{D}$ electronic spectra is described by a vibronic coupling that induces coherent energy exchange between excitons and quasi-resonant vibrations (see Supplementary Note 2 for further details):

$$
H_{e-v}=v_{1} \sqrt{S_{1}}\left(\left|3_{0}\right\rangle\left\langle 1_{1}|+| 1_{1}\right\rangle\left\langle 3_{0}\right|\right) .
$$

This implies that the vibronic coupling not only induces longlasting electronic excited-state coherences but also can mediate population transfer between excitonic bands. In a combination with thermal relaxation of exciton populations, the vibronic coupling may further enhance exciton population transfer and as a result could, in principle, have functional relevance in exciton transport ${ }^{14,38,43-45}$.

Interestingly, the different decoherence rates $\Gamma_{g 3} \approx 2 \Gamma_{g 1}$ of bands 1 and 3 lead to different amplitudes of the short-lived beating signals in N11 and R31 (Fig. 3b,c), which are determined by the prefactors $\Gamma_{g 1}^{-2}$ and $\Gamma_{g 3}^{-1} \Gamma_{g 1}^{-1}$, respectively. The lower decoherence rate of band 1 can be explained by band 1 being localized on the inner layer, while band 3 is delocalized over both the inner and outer layers ${ }^{46}$. As shown by the response functions for N11 and R31, the overall strength of the beating signals is proportional to the inverse of the electronic decoherence rates. It is therefore expected that the beating signal amplitude would diminish with an increase of the decoherence rate. This is the case for $\mathrm{N} 22$, where the physical situation in terms of excitonvibrational resonance $\left(\Delta \Omega_{32} \approx v_{2} \approx 470 \mathrm{~cm}^{-1}\right)$ is equivalent to $\mathrm{N} 11\left(\Delta \Omega_{31} \approx v_{1} \approx 668 \mathrm{~cm}^{-1}\right)$. The crucial difference is that band 2 has a higher decoherence rate than band 1 , as band 2 is localized on the outer layer exposed to solvent ${ }^{46}$. This explains the broader linewidth of band 2 in absorption and 2D spectra. Using an estimated value of $\Gamma_{g 2} \approx(47 \mathrm{fs})^{-1}$, the presented theory predicts the strength of N22 to be 5\% of N11 (see Supplementary Note 2), which is in line with the experimental observations (Fig. 2f). These results indicate that the experimentally observed long-lived beating signals, induced by vibronic mixing, require adequately low electronic decoherence rates, highlighting that resonance between exciton energy splitting and vibrational frequency alone is not sufficient ${ }^{47}$.

The presented vibronic model achieves quantitative agreement with the experimental observations. Crucially, the constraints imposed by the observed asymmetric decoherence rates $\Gamma_{g 3} \approx 2 \Gamma_{g 1}$ and fast relaxation of exciton population in $\mathrm{C} 8 \mathrm{O} 3$ on sub-picosecond timescales ${ }^{33}$ rule out incoherent models, where long-lived oscillations are sustained by Markovian correlated fluctuations (see Supplementary Note 3 for a detailed analysis). This further supports our conclusion that the observed experimental data provide evidence for vibronic mixing being the mechanism at play in our system.

We note that our results do not imply that correlated fluctuations can be universally ruled out, as this mechanism could be in place in certain pigment-protein complexes. The notion of correlated fluctuations has been developed for photosynthetic complexes where pigments are embedded in a protein scaffold. The protein has been considered as the potential source of correlated fluctuations in natural light harvesters ${ }^{16,17}$. For $\mathrm{C} 8 \mathrm{O} 3$, a structural frame such as a protein scaffold is absent and therefore correlated fluctuations are unlikely to induce longlived oscillatory 2D signals, which is in line with our observations.

\section{Discussion}

We have verified, theoretically and experimentally, that coherent vibronic coupling in the electronic excited-state manifold is responsible for the long-lived beating signals observed in $2 \mathrm{D}$ spectra of an artificial light harvester. The relatively simple electronic and vibrational structure of the investigated molecular aggregate along with its macroscopic alignment allowed us to rule out the presence of correlated fluctuations. The specific geometry of our system allowed us to gain further insights by illustrating the conditions under which intra-pigment vibrations can prolong electronic coherent effects. The moderately low decoherence rate of band 1, localized on the inner layer and protected from solvent, is the basis for exciton-vibrational coupling as the source of longlived beating signals. The outer band 2 , even though resonantly coupled to a vibration, exhibits a higher decoherence rate and therefore fails to produce observable oscillations. We conclude that the mere resonance between excitons and vibrations does not suffice to explain long-lived beating signals. An adequately low electronic decoherence rate, determined by the interaction between system and bath, is an equally important prerequisite.

The influence of vibronic coupling on energy transport in molecular aggregates has been extensively studied in the past, as recently reviewed ${ }^{44}$. The vibronic coupling has recently gained new interest (see ref. 48 for a recent tutorial overview), as it was suggested as a feasible mechanism to explain long-lived oscillations in the 2D spectra of several natural light-harvesting complexes and a photosynthetic reaction centre ${ }^{9,10}$. The requirement of exciton-vibrational resonance is readily satisfied in such systems, given their numerous excitonic bands and rich vibrational structures. Incoherent models based on correlated fluctuations were not ruled out though. Our work provides a quantum mechanical foundation for enhanced energy transfer based on vibronic coupling. As recently demonstrated, this mechanism is not limited to natural light harvesting, vibronic coupling is also of key importance in photovoltaic devices ${ }^{49}$.

\section{Methods}

Polarization-controlled 2D electronic spectroscopy. In 2D electronic spectroscopy, three ultrashort laser pulses generate an optical response of a molecular ensemble, which is spectrally resolved along both absorption $\left(\omega_{1}\right)$ and detection $\left(\omega_{3}\right)$ frequencies within the laser pulse spectrum. The absorption frequency $\omega_{1}$ is obtained by precise scanning of the time delay between the first two pulses and subsequent Fourier transformation $\left(t_{1} \rightarrow \omega_{1}\right)$. In detection, the signal is spectrally dispersed, leading directly to the detection frequency $\omega_{3}$. Varying time delay $t_{2}$ between pulses 2 and 3 provides information about evolution of the system on a femtosecond timescale ${ }^{50-52}$. To retrieve the purely absorptive part, the signal induced by pulses $1-3$ is detected in a heterodyned fashion by interfering it with a phase-stable local oscillator pulse. Polarization control is achieved by the combination of $\lambda / 4$ wave plates and wire grid polarizers for each of the laser beams to select the desired polarization with high accuracy. Polarization-resolved 2D experiments change the relative contributions of distinct pathways depending on 
the polarization of the laser pulses, orientation of the transition dipole moments and isotropy of the sample ${ }^{40}$. Rephasing spectra were acquired with a polarization sequence of $\left(90^{\circ}, 0^{\circ}, 90^{\circ}, 0^{\circ}\right)$ for pulses $(1,2,3$, local oscillator), in contrast to nonrephasing spectra, where the time ordering of the first two pulses is reversed, leading to a polarization sequence of $\left(0^{\circ}, 90^{\circ}, 90^{\circ}, 0^{\circ}\right)$. The polarization scheme used for rephasing spectra (Fig. 1d) shows $0^{\circ}$ was defined to be parallel to the sample flow direction, depicted as a white arrow in Fig. 1a. For a macroscopically aligned sample, this particular polarization sequence selects pathways stemming from interband coherences and vibronic mixing ${ }^{12,15}$, discussed throughout the paper, while pathways with all-parallel transition dipole moments such as groundstate bleach, stimulated emission, excited-state absorption and also vibrational wave packet excitation are suppressed. For the details regarding the experimental methods, see Supplementary Note 1. To subtract the non-oscillatory signals from 2D spectra, we employed a decay-associated spectra analysis ${ }^{33}$, where the population decays were fitted by a sum of three $2 \mathrm{D}$ spectra with individual decay constants. The $\omega_{2}$ maps in Fig. 2 were obtained using Fourier transformation $\left(t_{2} \rightarrow \omega_{2}\right)$ with zero-padding up to $2^{7}$ data points. All measurements were carried out at room temperature.

\section{References}

1. van Amerongen, H., Valkunas, L. \& van Grondelle, R. Photosynthetic Excitons (World Scientific, 2000).

2. Blankenship, R. E. Molecular Mechanisms of Photosynthesis (Blackwell Science, 2002).

3. Renger, T., May, V. \& Kühn, O. Ultrafast excitation energy transfer dynamics in photosynthetic pigment-protein complexes. Phys. Rep. 343, 137-254 (2001).

4. Huelga, S. F. \& Plenio, M. B. Vibrations, quanta and biology. Contemp. Phys. 54, 181-207 (2013).

5. Jonas, D. M. Two-dimensional femtosecond spectroscopy. Annu. Rev. Phys. Chem. 54, 425-463 (2003).

6. Engel, G. S. et al. Evidence for wavelike energy transfer through quantum coherence in photosynthetic systems. Nature 446, 782-786 (2007).

7. Dostál, J., Mančal, T., Vácha, F., Pšenčík, J. \& Zigmantas, D. Unraveling the nature of coherent beatings in chlorosomes. J. Chem. Phys. 140, 115103 (2014).

8. Collini, E. et al. Coherently wired light-harvesting in photosynthetic marine algae at ambient temperature. Nature 463, 644-647 (2010).

9. Romero, E. et al. Quantum coherence in photosynthesis for efficient solarenergy conversion. Nat. Phys. 10, 676-682 (2014).

10. Fuller, F. D. et al. Vibronic coherence in oxygenic photosynthesis. Nat. Chem. 6, 706-711 (2014)

11. Chin, A. W. et al. The role of non-equilibrium vibrational structures in electronic coherence and recoherence in pigment-protein complexes. Nat. Phys. 9, 113-118 (2013).

12. Plenio, M. B., Almeida, J. \& Huelga, S. F. Origin of long-lived oscillations in 2D-spectra of a quantum vibronic model: electronic versus vibrational coherence. J. Chem. Phys. 139, 235102 (2013).

13. Chin, A. W., Huelga, S. F. \& Plenio, M. B. Coherence and decoherence in biological system: principles of noise assisted transport and the origin of longlived coherences. Phil. Trans. R. Soc. A 370, 3638-3657 (2012).

14. Kolli, A., O’Reilly, E. J., Scholes, G. D. \& Olaya-Castro, A. The fundamental role of quantized vibrations in coherent light harvesting by cryptophyte algae. J. Chem. Phys. 137, 174109 (2012).

15. Tiwari, V., Peters, W. K. \& Jonas, D. M. Electronic resonance with anticorrelated pigment vibrations drives photosynthetic energy transfer outside the adiabatic framework. Proc. Natl Acad. Sci. USA 110, 1203-1208 (2013).

16. Lee, H., Cheng, Y.-C. \& Fleming, G. R. Coherence dynamics in photosynthesis: protein protection of excitonic coherence. Science 316, 1462-1465 (2007).

17. Ishizaki, A., Calhoun, T. R., Schlau-Cohen, G. S. \& Fleming, G. R. Quantum coherence and its interplay with protein environments in photosynthetic electronic energy transfer. Phys. Chem. Chem. Phys. 12, 7319-7337 (2010).

18. Hayes, D., Griffin, G. B. \& Engel, G. S. Engineering coherence among excited states in synthetic heterodimer systems. Science 340, 1431-1434 (2013).

19. Christensson, N. et al. High frequency vibrational modulations in twodimensional electronic spectra and their resemblance to electronic coherence signatures. J. Phys. Chem. B 115, 5383-5391 (2011).

20. Caycedo-Soler, F., Chin, A. W., Almeida, J., Huelga, S. F. \& Plenio, M. B. The nature of the low energy band of the Fenna-Matthews-Olson complex: vibronic signatures. J. Chem. Phys. 136, 155102 (2012).

21. Christensson, N., Kauffmann, H. F., Pullerits, T. \& Mančal, T. Origin of long-lived coherences in light-harvesting complexes. J. Phys. Chem. B 116, 7449-7454 (2012).

22. Heijs, D.-J., Dijkstra, A. G. \& Knoester, J. Ultrafast pump-probe spectroscopy of linear molecular aggregates: effects of exciton coherence and thermal dephasing. Chem. Phys. 341, 230-239 (2007).

23. Würthner, F., Kaiser, T. E. \& Saha-Möller, C. R. J-aggregates: from serendipitous discovery to supramolecular engineering of functional dye materials. Angew. Chem. Int. Ed. 50, 3376-3410 (2011).
24. Eisele, D. M. et al. Robust excitons inhabit soft supramolecular nanotubes. Proc. Natl Acad. Sci. USA 111, E3367-E3375 (2014).

25. Yuen-Zhou, J. et al. Coherent exciton dynamics in supramolecular lightharvesting nanotubes revealed by ultrafast quantum process tomography. ACS Nano 8, 5527-5534 (2014).

26. Qiao, Y. et al. Nanotubular J-aggregates and quantum dots coupled for efficient resonance excitation energy transfer. ACS Nano 9, 1552-1560 (2015).

27. von Berlepsch, H. \& Böttcher, C. in J-Aggregates Vol. 2 (ed. Kobayashi, T.) Ch. 4 119-153 (World Scientific, 2012).

28. von Berlepsch, H., Kirstein, S. \& Böttcher, C. Effect of alcohols on J-aggregation of a carbocyanine dye. Langmuir 18, 7699-7705 (2002).

29. von Berlepsch, H., Kirstein, S. \& Böttcher, C. Controlling the helicity of tubular J-aggregates by chiral alcohols. J. Phys. Chem. B 107, 9646-9654 (2003).

30. von Berlepsch, H. et al. Supramolecular structures of J-aggregates of carbocyanine dyes in solution. J. Phys. Chem. B 104, 5255-5262 (2000).

31. von Berlepsch, H. et al. Stabilization of individual tubular J-aggregates by poly(vinyl alcohol). J. Phys. Chem. B 107, 14176-14184 (2003).

32. Eisele, D. M. et al. Utilizing redox-chemistry to elucidate the nature of exciton transitions in supramolecular dye nanotubes. Nat. Chem. 4, 655-662 (2012).

33. Milota, F. et al. Vibronic and vibrational coherences in two-dimensional electronic spectra of supramolecular J-aggregates. J. Phys. Chem. A 117, 6007-6014 (2013).

34. Aydin, M., Dede, Ö. \& Akins, D. L. Density functional theory and Raman spectroscopy applied to structure and vibrational mode analysis of $1,1^{\prime}, 3,3^{\prime}$ tetraethyl-5,5',6,6'-tetrachloro-benzimidazolocarbocyanine iodide and its aggregate. J. Chem. Phys. 134, 064325 (2011).

35. Rich, C. C. \& McHale, J. L. Resonance Raman spectra of individual excitonically coupled chromophore aggregates. J. Phys. Chem. C 117, 10856-10865 (2013).

36. Butkus, V., Zigmantas, D., Abramavicius, D. \& Valkunas, L. Distinctive character of electronic and vibrational coherences in disordered molecular aggregates. Chem. Phys. Lett. 587, 93-98 (2013).

37. Womick, J. M. \& Moran, A. M. Exciton coherence and energy transport in the light-harvesting dimers of allophycocyanin. J. Phys. Chem. B 113, 15747-15759 (2009).

38. Womick, J. M. \& Moran, A. M. Vibronic enhancement of exciton sizes and energy transport in photosynthetic complexes. J. Phys. Chem. B 115, 1347-1356 (2011).

39. Del Rey, M., Chin, A. W., Huelga, S. F. \& Plenio, M. B. Exploiting structured environments for efficient energy transfer: the phonon antenna mechanism. J. Phys. Chem. Lett. 4, 903-907 (2013).

40. Hochstrasser, R. M. Two-dimensional IR-spectroscopy: polarization anisotropy effects. Chem. Phys. 266, 273-284 (2001).

41. Read, E. L. et al. Cross-peak-specific two-dimensional electronic spectroscopy Proc. Natl Acad. Sci.USA 104, 14203-14208 (2007)

42. Mukamel, S. Principles of Nonlinear Optical Spectroscopy (Oxford University Press, 1995).

43. Perlík, V. et al. Vibronic coupling explains the ultrafast carotenoid-tobacteriochlorophyll energy transfer in natural and artificial light harvesters. J. Chem. Phys. 142, 212434 (2015).

44. Schröter, M. et al. Exciton-vibrational coupling in the dynamics and spectroscopy of Frenkel excitons in molecular aggregates. Phys. Rep. 567, 1-78 (2015)

45. Killoran, N., Huelga, S. F. \& Plenio, M. B. Enhancing light-harvesting power with coherent vibrational interactions: a quantum heat engine picture. Preprint at http://arxiv.org/abs/1412.4136 (2015)

46. Didraga, C. et al. Structure, spectroscopy, and microscopic model of tubular carbocyanine dye aggregates. J. Phys. Chem. B 108, 14976-14985 (2004).

47. Halpin, A. et al. Two-dimensional spectroscopy of a molecular dimer unveils the effects of vibronic coupling on exciton coherences. Nat. Chem. 6, 196-201 (2014).

48. Chenu, A. \& Scholes, G. D. Coherence in energy transfer and photosynthesis. Annu. Rev. Phys. Chem. 66, 69-96 (2015).

49. Falke, S. M. et al. Coherent ultrafast charge transfer in an organic photovoltaic blend. Science 344, 1001-1005 (2014).

50. Brixner, T., Mančal, T., Stiopkin, I. V. \& Fleming, G. R. Phase-stabilized twodimensional electronic spectroscopy. J. Chem. Phys. 121, 4221-4236 (2004).

51. Augulis, R. \& Zigmantas, D. Two-dimensional electronic spectroscopy with double modulation lock-in detection: enhancement of sensitivity and noise resistance. Opt. Express 19, 13126-13133 (2011).

52. Augulis, R. \& Zigmantas, D. Detector and dispersive delay calibration issues in broadband 2D electronic spectroscopy. J. Opt. Soc. Am. B 30, 1770-1774 (2013).

\section{Acknowledgements}

We thank Valentyn I. Prokhorenko for help in 2D-DAS analysis. C.N.L. and J.H. acknowledge funding by the Austrian Science Fund (FWF): START project Y 631-N27 and by COST Action CM1202-PERSPECT-H2O. J.L., F.C.-S., S.F.H. and M.B.P. acknowledge funding by the EU STREP PAPETS and QUCHIP, the ERC Synergy Grant BioQ, the Deutsche Forschungsgemeinschaft (DFG) within the SFB/TRR21 and an 
Alexander von Humboldt Professorship. J.P. acknowledges funding by the Spanish Ministerio de Economía y Competitividad under Project No. FIS2012-30625. D.P. and D.Z. acknowledge funding by the Swedish Research Council and Knut and Alice Wallenberg Foundation.

\section{Author contributions}

D.P., D.Z. and J.H. designed and conducted experiments; H.v.B. was responsible for sample preparation, structural characterization and Raman measurements; J.L., F.C.-S., C.N.L., D.P., J.P. and J.H. analysed the data; J.L., F.C.-S., S.F.H., J.H. and M.B.P. developed theory; all authors discussed the results and wrote the manuscript.

\section{Additional information}

Supplementary Information accompanies this paper at http://www.nature.com/ naturecommunications
Competing financial interests: The authors declare no competing financial interests.

Reprints and permission information is available online at http://npg.nature.com/ reprintsandpermissions/

How to cite this article: Lim, J. et al. Vibronic origin of long-lived coherence in an artificial molecular light harvester. Nat. Commun. 6:7755 doi: 10.1038/ncomms8755 (2015).

\section{(c) (1)}

This work is licensed under a Creative Commons Attribution 4.0 International License. The images or other third party material in this article are included in the article's Creative Commons license, unless indicated otherwise in the credit line; if the material is not included under the Creative Commons license, users will need to obtain permission from the license holder to reproduce the material. To view a copy of this license, visit http://creativecommons.org/licenses/by/4.0/ 